\title{
Geometric Visualization Interaction System Based on Augmented Reality
}

\author{
Feng HAN 1,a, Xing-Qian LI 2,b* , Jian-Jun WANG ${ }^{3, c}$, Guan-Long QU ${ }^{4, d}$ \\ ${ }^{1}$ Mathematics School \& Institute of Jilin University, Changchun, Jilin University, Changchun \\ 130012,China \\ ${ }^{23}$ College of Computer Science and Technology, Jilin University, Changchun 130012,China \\ ${ }^{4}$ International School Beijing University of Posts and Telecommunications \\ a1071652171@qq.com, bSisyphusLi@foxmail.com, ‘616925652@qq.com, dtourista@bupt.edu.cn
}

\begin{abstract}
Keywords: augmented reality; immersive interaction; visualization; leap motion.
Abstract. With the continuous development of computer technology and CAD / CAM technology, people in the traditional manufacturing industry is no longer satisfied with the traditional machine tool design, so they now use the pop-ular 3D model printing technology. 3D printing technology could build geometric object model mold. In order to speed up the printing cycle and reduce the cost of model design, this paper proposes a geometric model visualiza-tion interaction system based on augmented reality. By using Leap Motion sensor to collect human arm and bone data, the arm movement path is calculated and processed. Path data for rapid sketch retrieval is matched with the database in preparation for the construction of three-dimensional objects. Then with the help of AR in-teraction, we could further design the mold. Experiments show that the system can make users quickly design and print out the model they want, with high stability, and can be highly efficient to help users to design geomet-ric model.
\end{abstract}

\section{Introduction}

The 3D printing model and other related models of the design process of a single, boring and other characteristics. To solve this problem, to improve the interaction of the model design process, so that the design more vivid. In this paper, the combination of virtual reality and enhance the reality of the combination of human-computer interaction technology applied to the geometric model design. Using Leap Motion capture gestures, Vuforia recognition, Oculus Rift multi-model data visualization display[1 4] Compared with the traditional interactive way, virtual reality and enhance the reality technology can simulate the virtual environment through electronic equipment, based on the user's behavior and three-dimensional real-time rendering of the dynamic environment interaction. Users can be immersed in a virtual reality through a virtual reality, the user to see and interact with all the objects are virtual structure. Virtual reality is the inevitable product of scientific and technological progress, human transformation of natural and thus simulate the reality, in adapting to the current technical conditions to improve the way people know the world. Enhance the reality of technology that is the combination of the actual situation, that is, want to add the object to enhance the real world, and thus interactive. Similarly, the user interacts with the object by capturing the natural operation of the human by accurately tracking the positioning device and the interactive device[5 6]. Enhance the reality of technology can be micro-macro and other world or details of the use of 3D animation simulation and display. Humans can carefully observe the internal nature of the object.

In order to solve the problem of single, boring and other problems in the 3D model design process, the interactive experience of 3D users in 3D geometric model design is more complicated and comfortable. At the same time, in order to speed up the design speed of geometric model, Fast model design reduces $3 \mathrm{D}$ printing cycles and reduces $3 \mathrm{D}$ printing costs. In this paper, the virtual reality and the enhancement of realistic technology as a system environment, through the Leap Motion module to capture the human arm and bone, do three-dimensional line path tracking, by allowing users to draw lines in the air, geometric model design, the three lines for sketch retrieval[7], And a similar approximation of the three-dimensional geometric model, and further preview the geometric model data visualization, projected in the enhanced reality equipment. The interactive system to increase the 
user in the 3D print or geometric model design of the interactive experience, creating a unique design fun, so that everyone can easily and quickly design their own and beautiful and complete 3D model.

\section{The Algorithm Framework of This Paper}

As shown in Figure 1, this algorithm includes the following steps: First, by using the Leap Motion module to detect gesture movement. Firstly, the hand motion data is captured in real time, and the current frame parameters are obtained. A series of processing is carried out by the feature transformation to obtain the displacement, normal vector, transformation direction and Euler angle change value of the gesture. The three-dimensional coordinate system is established, After simple processing to generate three-dimensional sketch. The feature is extracted from the sketch, and the extracted features are matched with the database. Finally, the 3D model is generated. Through the Vuforia recognition, Oculus interacts the data visualization to confirm whether the model is output after the user needs the model.

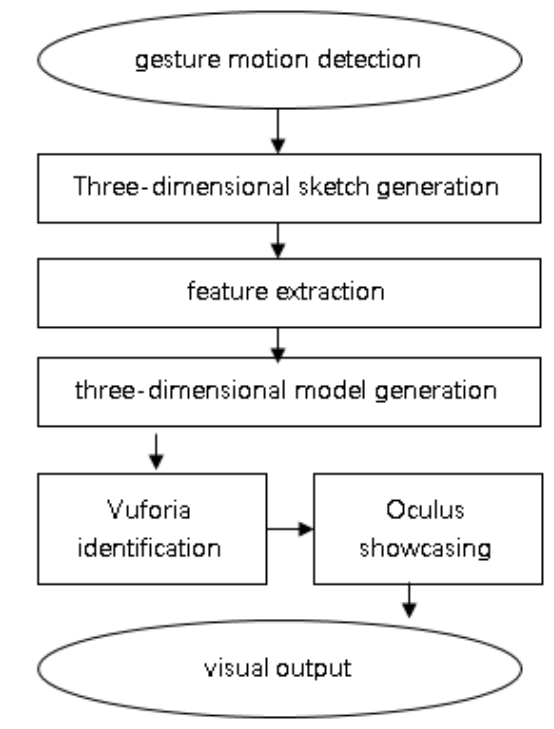

Fig. 1, algorithm framework

\section{To Enhance the Reality and Immersion Interaction}

\subsection{Input Modal Selection}

Compared to virtual reality equipment, most of the equipment in the way of interaction need to be pre-watched in this object, can trigger the relevant instructions and operations. There is a flaw in this way, and if you do not look at the object, you can not trigger an interactive command, such as an Oculus device. For the input mode, whether it is VR, AR or MR, but also to focus on the basis of interaction, supplemented by gestures or other interactive means, such as xbox, Touch, handle and other input devices. Speech recognition is another important interactive mode, but limited by its limited speech recognition interface, can not perform detailed and complex instructions, for most users is more cumbersome and tired. In summary, the current input mode still need to further quantify the convenience of the user, there is a large flaw for the interaction with the user.

Other devices such as Hololens and Kinect (s) to capture the depth of information in order to achieve the integration of realistic and deep information fusion, Leap Motion and Kinect for local capture accuracy of up to centimeters of gestures, and can track the body of mobile information, such as VR And Kinect (s) are used to assist in capturing the body in the virtual display scene to allow the user to see themselves or the other, as well as VR and Leap Motion, Kinect, Motion Capture to achieve the body of accurate information capture. 
Comparing the above input modes, this paper uses Leap Motion + Oculus Rift as the main interactive device. Leap Motion is a more popular somatosensory module. It captures the hand bone motion data in real time. It sends the serial data of the test data of the module to the PC through USB data line, and realizes the interaction between hand and $\mathrm{PC}$, and further retrieves the three-dimensional sketch. The input mode is simpler, more stable and reliable, and its way is easy to operate. Oculus rift is a virtual reality (VR) headset display. Through this device, the user can see its pre-set specific virtual world, through binocular parallax, produce a strong three-dimensional sense. In addition, since the Rift device is equipped with an inertial element such as a gyroscope, an accelerometer, etc., the user can perform a posture calculation in real time to analyze the Euler angle of the user's head, thereby determining the position of the user's head, The relevant angle. In this way, the user can feel a more realistic immersive virtual reality experience. But the existing Oculus Rift equipment can not accurately track the fine hand movements, if the use of Oculus Rift equipment alone, there are still flaws. Therefore, this paper presents a combination of Leap Motion module to capture the action of local finger joints to interact with the way, as shown in Figure 3. This approach, in conjunction with the advantages and disadvantages of the two devices, enables them to collaborate and expand the user's interactive experience, making the 3D geometric model design process more lively and more fun and improving the boring state of the usual designers.

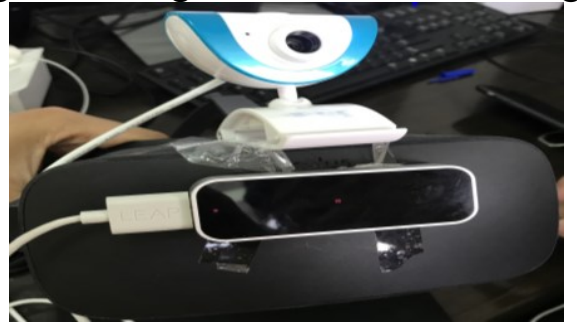

Fig. 2, Leap Motion+ Oculus Rift

\subsection{Vuforia Identification Technology}

Vuforia is the best development kit that combines virtual reality with the ability to complete recognition and accurate spatial tracking. $2 \mathrm{D}$ images and $3 \mathrm{D}$ objects can be identified after extracting feature points.

\subsubsection{Virtual Scene and Enhanced Scene Conversion}

In this paper, combined with Vuforia camera and Oculus camera work, can be directly used Oculus to identify. The advantage is that integration enhances reality with virtual reality. As shown in Figure 3.

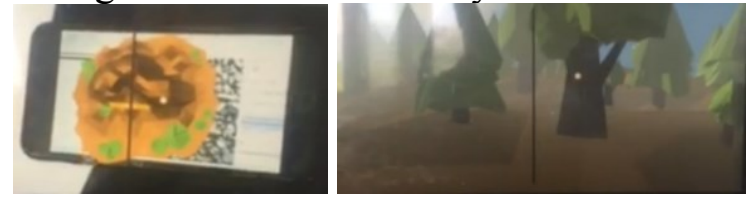

Fig. 3, Unity VR and AR

\subsubsection{Identify and Track at the same time}

The Image Targets module is supported in the Vuforia environment, which recognizes multiple 2D images. And Multi Targets can identify multiple Image Targets, which can track multiple simultaneous images at the same time. In the real environment, in order to enhance the authenticity of the user experience, often need to modify multiple design models at the same time, and the traditional screen-based design can not allow users to immerse the design of multiple three-dimensional model to display, so this article using Vuforia multi-model at the same time Identify tracing techniques to enhance their interactive authenticity. And the wearer can see the immersion of the content, the observer can also see, to facilitate the exchange of discussion, co-design. 


\subsubsection{Other Identification Objectives}

Cylinder Targets can be used to identify the packaging on 3D cylindrical objects. Usually used for commercial purposes. Text Recognition recognizes hundreds of thousands of English words. Object Recognition recognizes 3D objects. As shown in Figure 4.

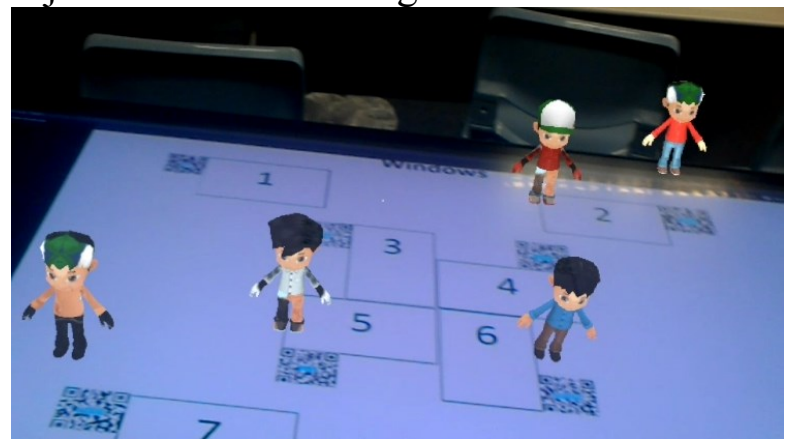

Fig. 4, Vuforia recognition recognizes

When the user in the three-dimensional geometric design of the fingers quickly move, the integration of virtual scenes and realistic scenes, on the basis of perception, through the image, gravity acceleration, depth information, gyroscope, voice recognition instructions, gesture instructions and other information can be achieved User authenticity immersive interaction.

\section{Geometric Model Retrieval Process}

\subsection{Generation of 3D Model Graphs}

The first step in the 3D model retrieval process is to generate a contour of its three-dimensional model based on a specific object, although one can express an object in a computer in detail by X-ray tomography, but it does not produce an object Dimensional contours of the objects are obtained by extracting the model characteristic lines. The two-dimensional contours need to be projected on the three-dimensional contours.

The characteristic line of the object model constitutes the three-dimensional feature map of the model, so the characteristic line of the object model is extracted first. The general method of extracting a characteristic line is to connect the curvature extreme point on the model with a line, which gives a detailed representation of the shape of the model.

In the actual $\mathrm{CAD}$ and computer graphics applications, the polygon mesh model is often used. The curvature information for the polygon mesh model is represented by the Laplace-Betrami operator. The curvature extremum points represent the size of the operator in the neighborhood of the mesh The

Laplace-Betrami operator:

$$
\Delta f\left(v_{i}\right)=\frac{1}{2 A_{i}} \sum_{v_{j} \in \mathrm{N}_{1}\left(v_{i}\right)}\left(\cot \alpha_{i, j}+\cot \beta_{i, j}\right)\left(f_{j}-f_{i}\right)
$$

\subsection{Generation of Two-Dimensional Sketches}

As the above just get the outline of the three-dimensional model, the need for objects from a certain direction to see the past two-dimensional shape, you can select a number of visual objects from around the object, extract three-dimensional contours tangent with different lines of sight, so You can get objects from different directions to see the two-dimensional sketch.

\subsection{Characteristics Extraction of 2D Sketch}

The three-dimensional model has been obtained above the two-dimensional sketch, the next step on the draft feature extraction has been the next match. Compared with the feature extraction of ordinary images, the sketch is not rich in color information, but by a combination of some lines of 
characteristic lines. There are many excellent features in image retrieval: color histogram features describe the proportion of different colors in the whole image, although it can not describe the specific location of color, but its extraction and operation are very simple, has been widely used; HOG characteristics through And the gradient direction histogram of the local area of the image is calculated and extracted from the local area, so the change of the image geometry is very invariant. The SIFT feature is the scale invariant feature transformation. By extracting the image in the image Feature points and their size and direction to match the image feature points, even if the image angle is changed, it does not affect the feature points. There are many other features, each feature has its own advantages. The Gabor transform divides the signal into many small time units, Fourier transform analysis for each time unit in order to determine the frequency at which the signal is present in the current time unit. Gabor wavelet can be sensitive to the edge of the image, which is one of the reasons for using him to extract the edge of the image.

\section{Experiment and System Test}

\subsection{Model Design Test}

In the Linux series operating system Ubuntu14.04 version, call OpenSSE example library, through the use of $\mathrm{C}++$ programming, call Open CV, Open GL class library, through the use of CMake batch compilation, in Ubuntu given gui window bin file executable permissions, Use Leap Motion as the input, install the Leap Motion For Linux driver, connect the Leap Motion module, place the hand in front of Leap Motion in the Gui drawing area window, move along the graphics track, and look at the right preview area. The database matches the results. The experimental results are shown in Fig5.

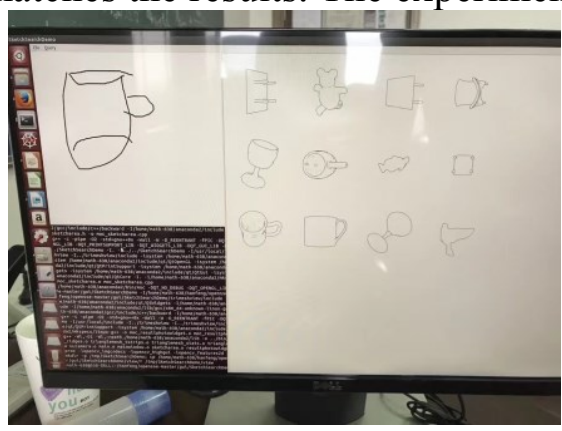

Fig. 5, sketches of the experiment

As shown in the figure above, through Leap Motion drawing the drawing in the Gui interface, the right view refreshes its database matching results in real time to help the user find the most suitable 3D model. In the experiment, the graph of the cup was drawn, and the right side showed all the results similar to the cup in real time for the user to pick. Experiments show that the three - dimensional sketch retrieval can meet its preset requirements, and has a high recognition rate. Leap motion is integrated with Oculus through Unity and can achieve the same effect.

\subsection{Geometric Visualization Interactive Testing}

In order to achieve immersive interaction, in the Unity set up inside the sky box, combined with Vuforia identification and Oculus equipment, through the Oculus above the installation of the camera, in Unity, which calls Open CV function to capture real-time video, presented in the sky box, so that in the virtual reality Equipment to achieve the effect of enhancing the reality, and use Vuforia identify key graphics, the previous step three-dimensional sketch retrieval under the three-dimensional geometric model visualization projection in the top of the key graphics, so that users get a good interactive experience. The experimental results are shown in Fig 6. 


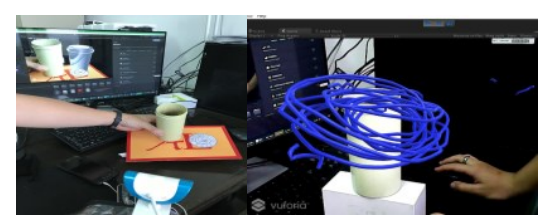

Fig. 6, geometric model projection display

As shown in the above figure, the visualization model of the three-dimensional cup sketch retrieved from the previous search based on this search method is projected on the key graphs of the experiment preset. And Vuforia identified the key graphics location, in order to enhance the reality of the design method described in the design of the cup model for visual projection display. And can continue to design lines on the virtual projection, immersive interaction with the user. Experiments show that the system has good and good interactive features, the virtual and realistic combination of the form for the user to display visual 3D model to help users quickly geometric model design.

\section{Summary and Conclusion}

This paper summarizes the 3D printing, virtual reality, and realizes the technical background. Aiming at the related methods and application of sketch retrieval, this paper proposes a method of immersive interactive design combined with Augmented Reality and Virtual Reality technology, which can be applied to manufacturing, Industrial fast geometric retrieval design system.

By analyzing and comparing the interactive input modes, this paper chooses the Leap Motion + Oculus Rift combination device to interact and capture the human trajectory of the human hand to further three-dimensional retrieval, and the 3D model is projected in the new geometric model design system of Oculus Rift equipment. Finally, Vuforia multi-object recognition technology is introduced, and its key theory is applied to the system to verify the correctness of its theory.

Experiments show that the system can quickly design and modify the three-dimensional model in the traditional model design software, and the interactive process is fun and vivid, it is easier to get started and the operation is simple. , The interaction process is more humane, the design process is more stable, reliable and efficient.

\section{References}

[1] Dictionary.com "virtual reality," in Collins English Dic-tionary - Complete \& Unabridged 10th Edition. Source location: Harper CollinsPublishers. Accessed: March 4, 2017.

[2] Steuer, Jonathan. Defining Virtual Reality: Dimensions Determining Telepresence,Department of Communication, Stanford University. 15 October 1993.

[3] P. Milgram and A. F. Kishino (1994)."Taxonomy of Mixed Reality Visual Displays".IEICE Transactions on Infor-mation and Systems. pp. 1321-1329. Retrieved 2013-10-17.

[4] Milgram P, Takemura H, Utsumi A, et al. Augmented reality: A class of displays on the reality-virtuality continu-um[C]/Photonics for industrial applications. International Society for Optics and Photonics, 1995: 282-292.

[5] Milgram P, Kishino F. A taxonomy of mixed reality visual displays[J]. IEICE TRANSACTIONS on Information and Systems, 1994, 77(12): 1321-1329.

[6] C. E. Jacobs, A. Finkelstein, and D.H.Salesin. Fast multi-resolution image querying.In ACM SIGGRAPH, Computer Graphics Proceedings, Annual Conference Series, Los Angeles, USA, 1995: 277 286.

[7] J. R. Smith and S.-F. Chang. VisualSEEk: a fully automated content-based image query system. Proceedings of the Fourth ACM International Conference on Multimedia, 1996: 87 98, Boston, USA. 